\title{
Efeito da extroversão e da atividade física no desempenho de habilidades motoras
}

http://dx.doi.org/10.11606/1807-5509201700040797

\author{
Natália Barros BELTRÃO* \\ Cássio De Miranda MEIRA JÚNIOR*** \\ Maria Teresa CATTUZZO***
}

\section{Resumo}

0 presente estudo teve como objetivo verificar a contribuição das variáveis traço de personalidade extroversão e nível de atividade física no desempenho de habilidades motoras. Crianças com idades entre 7-10 ( $n=80)$ foram avaliadas por meio do teste MABC. Um modelo de regressão foi proposto e incluiu extroversão, atividade física e gênero como variáveis de previsão para a pontuação total no teste e para os subtestes de destreza manual, controle de objetos e equilibrio. Os resultados indicaram que a extroversão foi uma variável significativa para a predição das habilidades de destreza manual $(p<0,01)$ e o desempenho motor total $(p=0,12)$. No entanto, não foi relevante para a predição de equilibrio $(p=0,435)$ ou de habilidades de controle de objetos $(p=0,145)$. 0 nível de atividade física não mostrou associação significativa com o desempenho motor. Gênero foi capaz de prever habilidades de controle de objetos, de equilíbrio e o desempenho motor geral. Os resultados deste estudo indicam que a extroversão media o desempenho motor, mas parece ter influências distintas, dependendo da subclasse da habilidade.

Palavras-chave: Atividade Motora; Criança; Desempenho Psicomotor; Personalidade.

\section{Introdução}

As diferenças individuais influenciam o desempenho de habilidades motoras ${ }^{1}$. Especificamente, as características pessoais dos praticantes podem influenciar o desempenho, seja de forma positiva ou negativa, dependendo da personalidade do sujeito ${ }^{2}$. Traços de personalidade referem-se aos aspectos internos e externos do caráter peculiar de uma pessoa que influenciam o comportamento em situaçóes cotidianas $^{3}$. As diferenças na personalidade pode levar os indivíduos a reagir de maneira diferente ao mesmo estímulo ou situação. Por isso, elas devem ser consideradas ao se planejar ou executar uma habilidade motora.

Vários estudos suportam a hipótese de que o traço extroversão afeta o desempenho motor. Tem sido verificado, por exemplo, que (a) extrovertidos mostram tempo de movimento mais rápido do que os introvertidos ${ }^{4-6}$; que (b) crianças extrovertidas cometem menos erros do que as introvertidas ao executar uma tarefa de lançamento de dardos de salāo ${ }^{7}$; que (c) extrovertidos mostram um maior número de respostas corretas do que os introvertidos quando se aumenta o nível de dificuldade da tarefa ${ }^{8}$, e que (d) introvertidos desempenhando tarefas de tempo de reação apresentam processamento prémotor mais rápido, enquanto os extrovertidos têm processamento motor mais rápido 5 .

O traço extroversáo tem sido associado ao nível de ativação cortical dos indivíduos, sendo os sujeitos introvertidos mais ativados (ou
*Departamento de Educação Física, Universidade Federal Rural de Pernambuco, Recife, PE, Brasil. ${ }^{*}$ Escola de Artes, Ciências e Humanidades, Universidade de São Paulo, São Paulo, SP Brasil.

${ }^{* * *}$ Escola Superior de Educação Física, Universidade de Pernambuco, Recife PE, Brasil. 
excitados) do que os extrovertidos ${ }^{9}$. Acreditase que esses níveis de excitação justificam os comportamentos típicos determinados por esse traço de personalidade. Extrovertidos são sociáveis, impulsivos, ativos, ousados, persistem em uma tarefa por períodos mais curtos, não são capazes de manter a atenção em tarefas por longos períodos (necessitam de quebras e trocas de atividades), precisam de excitação e assumir riscos. Por sua vez, os introvertidos são retraídos, reflexivos, calmos, inibidos, introspectivos, persistem por mais tempo em uma tarefa, mantêm a atenção em tarefas por longos períodos, pensam antes de agir, e preferem atividades caseiras ${ }^{10,11}$.

A ativação neurocortical baseia-se no aumento da atividade do sistema ativador reticular ascendente (SARA). Extrovertidos têm baixos níveis de atividade no circuito retículo-cortical, o que caracteriza um arousal infra-ativado, por isso procuram situaçóes e/ou contextos estimulantes para elevá-lo; introvertidos tem nível habitual de arousal hiperativado, o que os leva a procurarem ambientes calmos e poucos estimulantes ${ }^{10,12}$. Em outras palavras, para atingir níveis ideais de excitação, extrovertidos buscam fontes de estimulação excessiva, e introvertidos tentam evitar essas fontes. A relaçáo entre as características de personalidade e a ativação cortical é sustentada por estudo realizado no nível neurocomportamental de análise ${ }^{13}$.

De acordo com WrISBERG ${ }^{14}$, o desempenho motor está associado com um estado de excitação ótimo. Para ambos os introvertidos e extrovertidos, o desempenho mais eficiente ocorre quando eles são estimulados a esse nível ótimo de ativação. Considerando que o traço extroversão-introversão está ligado ao nível de ativação do sujeito, e que o desempenho motor é influenciado pelo nível de

\section{Método}

\section{Amostra}

Este é um estudo quantitativo, transversal e explicativo ${ }^{26}$, cuja amostra não probabilística foi composta por 80 crianças (41 meninos: $9,27 \pm 1,20$ anos; $17,54 \pm 2,84 \mathrm{~kg} / \mathrm{m}^{2} ; 39$ meninas: $8,65 \pm 1,08$ anos; $18,25 \pm 2,92 \mathrm{~kg} / \mathrm{m}^{2}$ ), estudantes de uma escola da cidade do Recife, PE. Foram incluídos todos os estudantes de 7 a 10 anos que tivessem ativação do praticante ${ }^{15}$, é possível, portanto, prever alguma relação entre essas duas variáveis.

Com exceção do estudo de CAmpbell et al. ${ }^{16}$, todos os demais estudos incluem na sua amostra apenas valores extremos do traço extroversão, excluindo os sujeitos que apresentam valores intermediários do traço. Entretanto, os traços de personalidade, assim como outras variáveis psicológicas, se distribuem como na curva de Gauss de distribuição normal, de forma que os valores extremos são menos frequentes e os valores centrais mais frequentes ${ }^{17}$. Assim, recomenda-se que as amostras incluam indivíduos com pontuações intermediárias para extroversão.

Paralelamente, alguns modelos teóricos ${ }^{18-21}$ e estudos empíricos ${ }^{22-25}$ têm mostrado que o desempenho motor está relacionado com os níveis de atividade física em crianças. Esta é uma relação cíclica: a competência motora adquirida por meio da prática da atividade física influencia positivamente o desempenho motor, e quanto mais competentes as crianças forem, mais elas tenderão a se envolverem em atividades físicas ${ }^{42}$. A atividade física é, portanto, uma variável importante a considerar quando se investiga o desempenho motor em crianças.

Especialmente na infância, quando ocorrem mudanças muito frequentes nos padróes motores, é importante identificar os aspectos que podem explicar as diferenças no desempenho motor. Assim, o objetivo deste estudo foi identificar se a extroversão e atividade física pode explicar o desempenho motor em crianças de sete a dez anos de idade. As hipóteses do presente estudo foram (a) com base em conceitos teóricos, extroversão influencia o desempenho motor, e (b) com base em evidências empíricas ${ }^{22-25}$, a atividade física influencia positivamente o desempenho motor.

autorização dos responsáveis por meio da assinatura do Termo de Consentimento Livre e Esclarecido, que não apresentassem impedimento físico e/ou perceptual para realização dos testes; que obtivessem pontuaçáo maior que 3 pontos na avaliação do traço de personalidade extroversão; e que participaram voluntariamente de todas as fases da coleta. O estudo foi aprovado pelo Comitê de Ética em Pesquisa Envolvendo Seres Humanos (CEP-UPE n. 039/11; 
CAAE - 0028.0.097.000-11) e foi conduzido de acordo com os padróes éticos reconhecidos.

\section{Instrumentos e procedimentos}

O desempenho motor das crianças foi medido pelo Movement Assessment Battery for Children Second Edition (MABC-2) ${ }^{27}$, o qual foi validado para a população brasileira ${ }^{28}$. O MABC-2 é um teste que avalia a competência motora por meio de oito habilidades motoras em três domínios: destreza manual, controle de objeto e equilíbrio, e é capaz de identificar crianças que apresentam dificuldade de movimento, por meio de percentis. Além disso, o teste avalia crianças de 4 a 12 anos de idade e; no presente estudo foi utilizada a faixa de 7 a 10 anos. Um único avaliador treinado (acima de $85 \%$ de concordância intra e entre avaliadores) foi responsável pela instrução e demonstração das tarefas do teste; em seguida, o participante realizava uma tentativa-ensaio e duas tentativas formais, as quais eram pontuadas. No presente estudo não foram utilizados os valores de percentis para a predição da dificuldade de movimento; a medida de desempenho foi o Escore Padrão (EP) para o desempenho total no teste, e para cada subteste (destreza manual, controle de objeto e equilíbrio).

Para avaliar o traço de personalidade extroversão, foram utilizadas as questóes relativas ao traço extroversão da Escala de Traços de Personalidade para Crianças (ETPC) ${ }^{29}$, que tem sido utilizado com crianças brasileiras ${ }^{7,30,31}$. Cada criança recebeu uma pontuação (em termos brutos e percentuais), de acordo com a distribuição do traço extroversão que caracteriza sua personalidade, e foi classificada segundo tal pontuação: 4 a $6=50 \%$ extrovertido, $7 \mathrm{e}$ $8=75 \%$ extrovertido, 9 e $10=+75 \%$ extrovertido.
A aplicaçáo do ETPC foi feita por profissional certificado em Psicologia. Para evitar disparidades de compreensão gramatical e contextual, o questionário foi aplicado em formato de entrevista, de modo que o psicólogo fazia as perguntas e a criança respondia sim ou não a cada uma delas.

A medida da atividade física foi feita por meio do instrumento Physical Activity Checklist Interview ${ }^{32}$, na versão validada para crianças brasileiras (Lista de Atividades Físicas - LAF), a qual mostrou boa confiabilidade para aferição da atividade física de crianças de sete a dez anos ${ }^{33}$. O procedimento de aplicação do teste incluía uma explicação inicial sobre o que seria perguntado à criança, e uma avaliação rápida do referencial da criança em relaçáo à capacidade de estimar o tempo. Em seguida, o avaliador questionava se a criança havia praticado cada uma das atividades citadas na lista de atividades físicas, se a resposta fosse positiva, era questionado o tempo despendido nessa atividade, $\mathrm{e}$ qual a percepção de esforço que a criança sentiu ao praticar tal atividade. A medida da atividade física foi dada pelo somatório em minutos do tempo gasto em atividades físicas de baixa, média e alta intensidade.

\section{Análise de dados}

A análise dos dados envolveu o uso de uma regressão linear múltipla, considerando os escores para o teste $\mathrm{MABC}$, traço extroversão e nível de atividade física. $\mathrm{O}$ gênero também foi incluído no modelo, considerando seu papel significativo em estudos anteriores que investigaram o desempenho motor em crianças $^{34,35}$. Para todas as análises foi utilizado o pacote estatístico SPSS (versão 10.0), e um nível de significância de 5\%.

\section{Resultados}

A TABELA 1 descreve os valores médios e de dispersão para o resultado do teste motor em cada um dos domínios. Os dados do teste motor, os meninos superaram as meninas em todos os subtestes e no Escore Padrão Total ( $ð$ Média $=8,44 ; \mathrm{DP}=2,67$; q Média $=6,97, \mathrm{DP}=2,12$ ).

O mesmo ocorreu para o tempo gasto em prática de atividade física: meninos foram mais ativos do que as meninas ( $ð$ Média $=123,83$; $\mathrm{DP}=78,16$; + Média 100,00, $\mathrm{DP}=71,20$ ).
Atividades físicas de média intensidade foram as mais praticadas entre meninos e meninas. A "caminhada" foi a atividade física mais frequente de acordo com o questionário, $85 \%$ das crianças relataram esta atividade. As atividades como "atividades ao ar livre", "esportes com raquete", "beisebol/softbol" e "ginástica olímpica" não foram citadas pelas crianças. Análises de frequência mostraram que $45 \%$ da amostra era "pouco ativa" e $55 \%$ era "suficientemente 
ativa", de acordo com os critérios de classificação propostos por STRONG et al..$^{36}$

A distribuição de frequência absoluta e relativa para os grupos evidenciou que metade das crianças $(56,3 \%)$ apresentaram-se como " $75 \%$ extrovertidos". O restante das crianças estava distribuído no grupo de " $50 \%$ extrovertido" $(27,5 \%)$ e "mais do que 75\% extrovertido" (16,3\%).

TABELA 1 - Valores de média e desvio padrão (DP) para os subtestes do Movement Assessment Battery for Children - Second Edition, estratificado por sexo e para a amostra total.

\begin{tabular}{lccc}
\hline & $\begin{array}{c}\text { Meninos (n=41) } \\
\text { Média (DP) }\end{array}$ & $\begin{array}{c}\text { Meninas (n=39) } \\
\text { Média (DP) }\end{array}$ & $\begin{array}{c}\text { Total (n=80) } \\
\text { Média (DP) }\end{array}$ \\
\hline Escore Padrão (pontos) & & & \\
\hline Destreza manual & $7,95(3,26)$ & $7,72(2,96)$ & $7,84(3,10)$ \\
\hline Controle de Objetos & $11,17(2,55)$ & $9,28(2,14)$ & $10,25(2,53)$ \\
\hline Equilíbrio & $8,2(2,83)$ & $6,72(2,77)$ & $7,48(2,88)$ \\
\hline Total & $8,44(2,67)$ & $6,97(2,12)$ & $7,73(2,52)$ \\
\hline
\end{tabular}

Foi realizada uma análise de regressão múltipla incluindo extroversão, atividade física e gênero como variáveis dependentes. As variáveis foram adicionadas simultaneamente no modelo de regressão. Cada variável do teste de motor (EPT, EPDM, EPCO e EPE) foi testada separadamente enquanto variável independente. A TABELA 2 mostra os parâmetros ( $\beta$ e p) do modelo. Embora não tenha havido significância em relação à EPE, as equaçôes de regressão explicaram uma parcela significativa da variabilidade de EPT em termos de variabilidade em extroversão e sexo $\left[\mathrm{EPT}=4,14+(1,04 *\right.$ extroversão $)+\left(1,41^{*}\right.$ género $)$; $\left.\mathrm{R}^{2}=0,16(\mathrm{p}=0,004) ; \mathrm{F}_{(3,76)}=4,83 ; \mathrm{p}=0,004\right], \mathrm{em}$ EPMD em termos de variabilidade em extroversão $[$ EPMD = 3,92 + $(1,48 *$ extroversão $) ;$ $\left.\mathrm{R}^{2}=0,11(\mathrm{p}=0,031) ; \mathrm{F}_{(3,76)}=3,13 ; \mathrm{p}=0,031\right]$, e, EPCO em termos de variabilidade em gênero $\left[\mathrm{EPCO}=7,54+(1,83 *\right.$ gênero $) ; \mathrm{R}^{2}=0,16$ $\left.(\mathrm{p}=0,003) ; \mathrm{F}_{(3,76)}=5,02 ; \mathrm{p}=0,003\right]$.

TABELA 2 - Valores de beta normalizado $(\beta)$ e significância de cada variável independente (traço extroversão, atividade física e sexo), no escore padrão do desempenho motor total e em cada subteste do Movement Assessment Battery for Children - Second Edition.

Desempenho Motor (Escore Padráo)

\begin{tabular}{|c|c|c|c|c|c|c|c|c|}
\hline \multirow{2}{*}{ Variáveis independentes } & \multicolumn{2}{|c|}{ Destreza Manual } & \multicolumn{2}{|c|}{ Controle de Objetos } & \multicolumn{2}{|c|}{ Equilíbrio } & \multicolumn{2}{|c|}{ Total } \\
\hline & $\beta$ & $\mathbf{p}$ & $\beta$ & $\mathbf{p}$ & $\beta$ & $\mathbf{p}$ & $\beta$ & $\mathbf{p}$ \\
\hline Traço Extroversão & 0,314 & $0,005^{*}$ & 0,155 & 0,145 & 0,087 & 0,435 & 0,271 & $0,012^{*}$ \\
\hline Atividade Física & $-0,102$ & 0,354 & 0,015 & 0,891 & $-0,026$ & 0,813 & $-0,036$ & 0,738 \\
\hline Sexo & 0,035 & 0,753 & 0,364 & $0,001^{*}$ & 0,257 & $0,025^{*}$ & 0,282 & $0,012^{*}$ \\
\hline
\end{tabular}

\section{Discussão}

O objetivo do presente estudo foi identificar se o traço de personalidade extroversão e atividade física poderiam explicar o desempenho motor grosso em crianças. Postulava-se que a extroversão e atividade física teriam uma influência positiva sobre o desempenho do motor. No entanto, de acordo com nossos resultados, extroversáo parece ter uma influência distinta sobre certas categorias de movimento e atividade física náo é relevante para prever o desempenho motor. 
É relevante considerar que estamos investigando um comportamento multicausal, influenciado por fatores de diferentes naturezas. Segundo TANi et al. ${ }^{37}$, uma abordagem teórica fundamentada nas características integrativa e complexa dos sistemas, não mais focaliza a influência de um único fator sobre a organização do sistema neuromotor. Sobre isso, Thelen ${ }^{38}$ considera que direcionar a atençáo dos pesquisadores para a multicausalidade das açóes é fundamental para se compreender os padrôes de movimento. Nessa perspectiva, o fenômeno do desenvolvimento motor, não é causado por este ou aquele fator, mas por uma infinidade de elementos que interagem entre si. Sendo assim, os pesos das variáveis investigadas no presente estudo representam, mesmo que em baixas proporçóes, a possibilidade de previsibilidade de um comportamento que é influenciado por vários fatores simultaneamente.

No modelo de regressão, a extroversão esteve associada com o desempenho total de habilidades motoras e com as habilidades de destreza manual. Isto confirma parcialmente a hipótese inicial de que o desempenho motor é influenciado pela extroversão. De acordo com o modelo, a relaçáo entre extroversão e destreza manual é diretamente proporcional. Neste caso, se observarmos as características das tarefas de destreza manual, verificamos que apesar de serem tarefas que exigiam controle motor fino e precisão nos movimentos, a maioria delas exigia que os sujeitos executassem no menor tempo possível, e a medida de desempenho era o tempo em segundos.

A maior parte dos estudos que investigaram o traço extroversão e o desempenho motor explorou as diferenças provocadas por esse traço sobre o tempo de reação (TR) e tempo de movimento (TM). De acordo com esses estudos, sujeitos que pontuam em escores mais altos de extroversão apresentam TM menor, ou seja, são executores mais velozes ${ }^{4,6}$. Corroborando os achados desses estudos, nossos sujeitos mais extrovertidos foram os mais velozes nas tarefas de destreza manual. Outra fonte de apoio para os nossos resultados é a constatação de que, quando o tempo de resposta é controlado externamente, extrovertidos superaram os introvertidos ${ }^{8}$. Sendo assim, acredita-se que a restriçáo de tempo é um elemento fundamental para a relação proporcional entre extroversão e destreza manual.

Sujeitos extrovertidos, por terem nível de ativação mais baixo, procuram contextos estimulantes, como situaçóes esportivas grupais (esportes em equipe) e/ou que envolvem competitividade ${ }^{10} \mathrm{e}$ tais atividades esportivas envolvem, tipicamente, habilidades motoras grossas. Por isso, foi hipotetizado que os sujeitos mais extrovertidos desempenhariam melhor habilidades motoras grossas do que aqueles com menores pontos nesse traço. Entretanto, isso não ocorreu: o traço extroversão não exerceu qualquer influência sobre o desempenho em habilidades grossas de controle de objetos e de equilíbrio.

Apesar de já ser claro que diferentes escores em traços de personalidade provocam diferenças comportamentais, é importante ressaltar que a maioria das investigaçôes é baseada em comportamentos adultos. Sobre as crianças, sabese que elas passam, ao longo da infância, por um processo de desenvolvimento cognitivo, que as permite desenvolver conceitos mais realistas e complexos de si mesmos ${ }^{39}$. Mas esse é um processo contínuo, e apenas inicia-se na infância. Portanto, dos sete aos 10 anos a criança ainda se encontra nessa fase de aprender a se reconhecer, e apesar de ela já ser capaz de distinguir e avaliar suas atitudes, ela ainda está desenvolvendo o conceito do $\mathrm{eu}^{40}$.

Ao final da infância, a noção de autoconhecimento vai se ampliando e o relato de seus hábitos se torna mais fiel ao real ${ }^{39}$; ao mesmo tempo, os traços de personalidade vão se tornando mais estabilizados, e a personalidade vai se consolidando, até atingir a completa solidificação na fase adulta ${ }^{41}$. Pode-se supor, portanto, que na fase da infância, quando está ocorrendo o período de consolidação das características de personalidade, os traços podem não ser suficientemente estáveis para influenciar o desempenho motor grosso das crianças.

De acordo com RowLAND ${ }^{42}$ as crianças são inerentemente ativas, pois o movimento oferece a informação que o sistema nervoso central delas precisa para ser estimulado. Segundo essa ideia, todas as crianças fazem uso exclusivamente do movimento para regular seus níveis de ativação (ou arousal). O movimento representa uma necessidade de qualquer criança, pois ele configura-se um meio concreto que facilita a interação com o ambiente ${ }^{43}$. Independente dos níveis de ativação, crianças farão uso dos movimentos, pois eles são a sua forma de expressão e comunicação com o meio. Pode-se pensar, portanto, que nessa fase da vida, as diferenciações entre níveis de ativação, não sejam fortes e influentes o suficiente ao ponto de provocar efeitos sobre o movimento, especificamente no desempenho de habilidades motoras grossas. E assim, é possível pensar também que ao investigar crianças mais 
velhas, ou adultos, o traço possa influenciar de forma mais evidente o desempenho motor.

A atividade física não mostrou qualquer associação com o desempenho motor. Esse resultado contradiz os achados da literatura ${ }^{22-25,44}$. Essa falta de associação entre as variáveis podem estar ligadas ao tempo gasto em atividades físicas. De fato, se a amostra apresentasse baixos níveis de atividade física, era provável que a essa variável tivesse pouca expressão no desempenho motor. Entretanto, as análises descritivas mostraram que mais da metade das crianças atingiram níveis satisfatórios, de acordo com as recomendações dos Centros de Controle de Prevenção de Doenças (Centers of Disease Control and Prevention $)^{36}$. Assim, parece que a falta de associação entre nível de atividade física e desempenho motor não está ligada ao tempo gasto em atividades físicas, mas para o tipo de atividade que eles as crianças praticavam.

Outro aspecto a ser considerado é o tipo de medida e instrumento utilizado para medir a atividade física. Analisando os estudos que exploraram a relação entre atividade física e desempenho motor, observa-se que todos eles utilizaram medidas diretas para mensurar a atividade das crianças (pedômetros ou acelerômetros). Os resultados dessas investigaçóes mostraram que há correlação significativa entre essas variáveis, entretanto os valores dessas correlaçôes foram fracos ou moderados (valor de $r$ variando entre 0,34 e 0,59$)^{22,24,44}$. Isso sugere que medidas quantitativas de volume e/ou intensidade podem oferecer informação limitada para as investigaçóes que pretendem explorar as habilidades motoras. De acordo com Clark ${ }^{18}$, existe diferença entre atividades físicas e atividades que promovam desenvolvimento de habilidades motoras. De fato, conceitualmente, atividade física refere-se a qualquer movimento que produz contração muscular e gasto energético ${ }^{45}$; e, portanto, qualquer atividade enquadrada nesse contexto será referida como atividade física, independente se ela contribui ou não para o domínio motor da criança. O desenvolvimento do comportamento motor habilidoso depende da quantidade e da qualidade da prática, de forma que o processo de experimentação de habilidades motoras e exploraçáo das diversas formas de combinações das habilidades determinarão a competência motora ${ }^{46}$.

No presente estudo, numa tentativa de conhecer as atividades mais e menos praticadas, foi descrita a quantidade de crianças que praticaram cada uma das atividades físicas listadas no instrumento de atividade física (Lista de Atividades Físicas). A atividade mais praticada pelas crianças foi a caminhada (85\%), que é uma atividade caracterizada como de baixa complexidade efetora ou decisória, pouco desafiante para o sistema neuromotor. Se as crianças da amostra por um lado atingiram as recomendaçóes quanto à quantidade de atividade física praticada, a análise da natureza dessa atividade sugere que a atividade física mais praticada pelas crianças pouco deve contribuir para a diversidade e complexidade do repertório motor delas. Esses dados parecem, portanto, ratificar a ideia de que o fator quantidade pode náo ser tão relevante quanto o tipo (a qualidade) da atividade praticada, quando se considera o desenvolvimento das habilidades motoras.

O método de observação direta, desenvolvido especificamente para mensurar atividade física de crianças $^{47}$, parece se aproximar mais de uma abordagem que permita conhecer a natureza, as características ou os tipos de movimentos envolvidos nas atividades físicas. Tendo em vista que não existem instrumentos que permitam observação direta com mensuração individualizada e com detalhes qualitativos das habilidades envolvidas nas atividades praticadas pela criança, estudos que explorassem a validação de método deste tipo seriam relevantes para a área do comportamento motor.

Em relaçáo à variável gênero, embora ela não tenha sido uma variável principal do estudo, as análises mostraram que ela foi relevante para explicar o desempenho em habilidades de controle de objetos e de equilíbrio. Esse resultado está de acordo com estudos anteriores envolvendo habilidades de controle de objetos $^{34,48-53}$ e de equilíbrio ${ }^{35}$.

Por fim, considera-se que a utilizaçáo de um instrumento indireto para medir a atividade física, e o desenho transversal limitam as conclusóes deste estudo. Além disso, acredita-se que a inclusão de sujeitos mais velhos possa elucidar de forma mais clara a influência do traço de personalidade sobre o desempenho motor. Por isso, sugere-se que estudos futuros sejam conduzidos com crianças mais velhas ou adultos, utilizando observação direta da atividade física, e que as modificações no desempenho motor sejam acompanhadas em um protocolo longitudinal.

Os resultados deste estudo indicam que a extroversão media desempenho motor, mas parece ter influências distintas, dependendo da categoria de movimento. Ela é uma variável importante para a 
predição da destreza manual e do desempenho motor geral. No entanto, extroversão não foi relevante na predição de habilidades de equilíbrio ou de controle de objetos. Além disso, enquanto o nível de atividade física não foi significativamente associada com o desempenho do motor, o gênero foi capaz de predizer o desempenho me habilidade de equilíbrio, controle de objetos e o desempenho motor geral.

\section{Abstract}

\section{Effect of extroversion and physical activity on motor skills performance}

This study aimed to verify the contribution of the variables extraversion personality trait and physical activity level on the performance of motor skills. 80 children aged 7-10 performed the MABC test. A regression model was proposed and included extraversion, physical activity and gender as predictor variables for the total test score and for the subtests of manual dexterity, object control and balance. The results indicated that extraversion was a significant variable for predicting manual dexterity skills $(p<0.01)$ and the total motor performance $(p=0.12)$. However, it was not relevant for predicting balance $(p=0.435)$ or object control skills $(p=0.145)$. The level of physical activity showed no significant association with motor performance. Gender was able to predict object control skills, balance and total motor performance. The results of this study indicate that extroversion does mediate motor performance, but it seems to have distinct influences, depending on the subclass skill.

KeYwords: Motor Activity; Child; Psychomotor Performance; Personality.

\section{Referências}

1. Magill RA. Motor learning and control: concepts and applications. 9a ed. New York: Mcraw Hill; 2011.

2. Weinberg RS, Gould D. Foundations of sport and exercise psychology. Champaign: Human Kinetics; 2011.

3. Schultz DP, Schultz SE. Teorias da personalidade. São Paulo: Pioneira Thomson; 2002.

4. Doucet C, Stelmack RM. Movement time differentiates extraverts from introverts. Pers Indiv Differ. 1997;23(5):775-86.

5. Rammsayer T, Stahl J. Extraversion-related differences in response organization: evidence from lateralized readiness potentials. Biol Psychol. 2004;66(1):35-49.

6. Stelmack RM, Houlihan M, McGarry-Roberts PA. Personality, reaction time, and event-related potentials. J Pers Soc Psychol. 1993;65(2):399-409.

7. Meira Junior CM, Perez CR, Maia RF, Neiva JFO, Barrocal RM. Extroversão, neuroticismo e desempenho motor em crianças executando arremessos de dardo de saláo. Rev Bras Psicol Esp. 2008;2(1):1-14.

8. Kirkcaldy DK. Influence of personality variables on performance. Percept Mot Skills. 1984;58(2):640-2.

9. Eysenck HJ. The biological basis of personality. New Brunswick: Transaction Publishers; 2006.

10. Colom R, Flores-Mendoza C. Introdução à psicologia das diferenças individuais. Porto Alegre: ArtMed; 2006.

11. Matthews G, Deary IJ, Whiteman MC. Personality traits. Cambridge: Cambridge University Press; 2003.

12. Schmidt V. Las bases biológicas del neuroticismo y la extraversión. Rev Psiencia. 2010;2(1):20-5.

13. Kumari V, Gray JA, Mitterschiffthaler MT, Das M, Zachariah E, Vythelingum GN, et al. Cognitive effects of nicotine in humans: an fMRI study. Neuroimage. 2003;19(3):1002-13.

14. Wrisberg CA. The arousal-performance relationship. Quest. 1994;46(1):60-77.

15. Schmidt RA, Wrisberg CA. Aprendizagem e performance motora. 4a ed. Porto Alegre: 2010. 416 p.

16. Campbell AM, Davalos DB, McCabe DP, Troup LJ. Executive functions and extraversion. Pers Indiv Differ. 2011;51(6):720-5.

17. Chamorro-Premuzic T. Personality and individual differences. Malden: Wiley-Blackwell; 2007.

18. Clark JE. On the problem of motor skill development. J Phys Educ Recreat Dance. 2007;78(5):1-58.

19. Clark JE. From the beginning: a developmental perspective on movement and mobility. Quest. 2005;57(1):37-45.

20. Goodway J. What's skill got to do with it? A developmental approach to promoting physical activity in urban youths. J Phys Educ Recreat Dance. 2009;80(8):38-40. 
21. Stodden DF, Goodway JD, Langendorfer SJ, Roberton MA, Rudisill ME, Garcia C, et al. A developmental perspective on the role of motor skill competence in physical activity: an emergent relationship. Quest. 2008;60(2):290-306.

22. Fontana F, Mazzardo O, Gallagher J. The correlation between fundamental movement skills and step count in second grade children. Med Sci Sports Exerc. 2008;40(5):S411.

23. Livesey D, Lum Mow M, Toshack T, Zheng Y. The relationship between motor performance and peer relations in 9- to-12year-old children. Child Care Health Dev. 2011;37(4):581-8.

24. Mazzardo O. The relationship of fundamental movement skills and level of physical activity in second grade children. Pittsburgh: University Of Pittsburgh; 2008.

25. Ziviani J, Poulsen A, Hansen C. Movement skills proficiency and physical activity: a case for Engaging and Coaching for Health (EACH): child. Aust Occup Ther J. 2009;56(4):259-65.

26. Sampieri RH, Collado CH, Lucio PB. Metodologia de pesquisa. 3 ed. ed. São Paulo: McGraw-Hill; 2006.

27. Henderson S, Sugden DA, Barnett A. Movement assessment battery for children. 2a ed. San Antonio: Pearson; 2007.

28. Valentini N, Ramalho M, Oliveira M. Movement assessment battery for children 2: translation, reliability, and validity for Brazilian children. Res Dev Disabil. 2014;35(3):733-40.

29. Sisto FF. Escala de traços de personalidade para crianças: ETPC. São Paulo: Vetor; 2004.

30. Sisto FF, Oliveira SMS, de Oliveira KL, Bartholomeu D, Oliveira JCS, Costa ORS. Escala de traços de personalidade para crianças e aceitação social entre pares. Interação em Psicologia. 2004;8(1):15-24.

31. Sisto FF, Rueda FJM. Estudo sobre as relaçóes entre autocontrole e traços de personalidade. Psicol Esc Educ. 2008;12(2):369-80.

32. Sallis JF, Strikmiller PK, Harsha DW, Feldman HA, Ehlinger S, Stone EJ, et al. Validation of interviewer-and self-administered physical activity checklists for fifth grade students. Med Sci Sports Exerc. 1996;28(7):840-51.

33. Adami F, Cruciani F, Douek M, Sewell CD, Mariath AB, Hinnig PF, et al. Confiabilidade do questionário Lista de Atividades Físicas em crianças. Rev Saúde Públ. 2011;45(2):321-33.

34. Carvalhal M, Vasconcelos-Raposo J. Diferenças entre gêneros nas habilidades: correr, saltar, lançar e chutar. Rev Motricidade. 2007;3(3):44-56.

35. Paim MCC. Desenvolvimento motor de crianças pré-escolares entre 5 e 6 anos. Revista Digital Buenos Aires. 2003;8(58):1-11.

36. Strong WB, Malina RM, Blimkie CJR, Daniels SR, Dishman RK, Gutin B, et al. Evidence based physical activity for schoolage youth. J Pediatr. 2005;146(6):732-7.

37. Tani G, Meira Júnior CM, Ugrinowitsch H, Benda RN, Chiviacowsky S, Corrêa UC. Pesquisa na área de comportamento motor: modelos teóricos, métodos de investigação, instrumentos de análise, desafios, tendências e perspectivas. Rev Educ Fís. 2010;21(3):329-80.

38. Thelen E. Motor development: a new synthesis. Am Psychol. 1995;50(2):79-95.

39. Papalia D, Olds S. Motor development. Porto Alegre: Artes Médicas; 2000.

40. Jung CG, Amaral V. O desenvolvimento da personalidade. Petrópolis: Vozes; 1986.

41. Pervin LA, John OP. Personalidade: teoria e pesquisa. 8a ed. Porto Alegre: Artmed; 2004.

42. Rowland TW. The biological basis of physical activity. Med Sci Sports Exerc. 1998;30(3):392-9.

43. Eaton WO, McKeen NA, Campbell DW. The waxing and waning of movement: implications for psychological development. Dev Rev. 2001;21(2):205-23.

44. Lopes LO, Lopes VP, Santos R, Pereira BO. Associaçôes entre actividade física, habilidades e coordenação motora em crianças portuguesas. Rev Bras Cineantropom Desempenho Hum. 2011;13(1):15-21.

45. ACSM. Diretrizes do ACSM para os testes de esforço e sua prescrição. 7a ed. Rio de Janeiro: Guanabara Koogan; 2007.286 p.

46. Cattuzzo MT, Beltrão NB, Campos MCC, Guerra ERF. A multicausalidade desenvolvimental: uma abordagem sistêmica da proficiência motora e da prática da atividade física. In: Cattuzzo MT, Caminha IO, editores. Fazer e pensar ciência em educação física: livro 1. João Pessoa: Editora Universitária da UFBP; 2012.

47. Welk GJ, Corbin CB, Dale D. Measurement issues in the assessment of physical activity in children. Res Q Exerc Sport. 2000;71(2 supl):S59-73.

48. Afonso GH, Freitas DL, Carmo JM, Lefevre JA, Almeida MJ, Lopes VP, et al. Desempenho motor: um estudo normativo e criterial em crianças da Regiāo Autónoma da Madeira, Portugal. Rev Port Cien Desp. 2009;9(2-3):160-74.

49. Barnett LM, van Beurden E, Morgan PJ, Brooks LO, Beard JR. Does childhood motor skill proficiency predict adolescent fitness? Med Sci Sports Exerc. 2008;40(12):2137-44.

50. Spessato BC. Trajetórias de desenvolvimento motor de crianças e o engajamento em uma proposta interventiva inclusiva para maestria [dissertação]. Porto Alegre: Universidade Federal do Rio Grande do Sul; 2009. 
51. Villwock G, Valentini NC. Percepção de competência atlética, orientação motivacional e competência motora em crianças de escolas públicas: estudo desenvolvimentista e correlacional. Rev Bras Educ Fís Esporte. 2007;21(4):245-57.

52. Villwock GMC. O estudo desenvolvimentista da percepção de competência atlética, da orientação motivacional, da competência motora e suas relaçóes em crianças de escolas públicas [dissertação]. Porto Alegre: Universidade Federal do Rio Grande do Sul; 2005.

53. Valentini NC. Percepçóes de competência e desenvolvimento motor de meninos e meninas: um estudo transversal. Movimento (ESEF/UFRGS). 2002;8(2):51-62.

\begin{tabular}{|c|c|}
\hline $\begin{array}{r}\text { ENDEREÇo } \\
\text { Natália Barros Beltrão } \\
\text { o de Educação Física da } \\
\text { l Rural de Pernambuco } \\
\text { iros, s/n - Dois Irmãos } \\
\text { - Recife - PE - BRASIL } \\
\text { il.com; nataliabeltrao@ } \\
\text { defis.ufrpe.br }\end{array}$ & $\begin{array}{l}\text { Recebido para publicação: 19/07/2015 } \\
\text { Revisado: 03/11/2016 } \\
\text { Aceito: 15/12/2016 }\end{array}$ \\
\hline
\end{tabular}

